Nano LIFE

Vol. 1, Nos. 3 \& 4 (2010) iii-iv

(C) World Scientific Publishing Company

DOI: $10.1142 /$ S1793984410000456

\title{
NANOMEDICINE: THERAPEUTICS, DIAGNOSTICS, AND IMAGING AGENTS
}

In the last couple of decades, the fields of engineering, cell biology, chemistry and physics have experienced a renaissance through the rise of nanotechnology, which has facilitated greater interactions among these fields. As a result, nanomedicine emerged - the multidisciplinary field encompassing principles from nanotechnology which directly impacts biomedical sciences. Nanomedicine opens the door to the generation of advanced drug delivery systems, personalized theranostics, and medical imaging at the molecular level for differed diseases. Numerous nanoscale therapeutic and imaging agents (e.g. liposomaland albumin-based nanoparticles, Gd-DTPA, ferumoxides) have received approval for clinical use in humans worldwide and have already provided clinical benefit. This special issue of Nano LIFE brings to forth the latest developments in nanomedicine research focusing on cutting-edge developments in diagnostic systems, therapeutics and drug delivery, and imaging agents.

One of the two diagnostic systems discussed in this issue analyzes the role of nanoconfinement in nanoporous membranes and explores approaches to design sensitive molecular biosensors for the enhanced detection of cardiovascular biomarker proteins. In particular, Vattipalli et al. describe their approach to exploit the perturbation of the electrical double layer of metallic microscale sensing sites upon protein binding in a high density sandwiched array of nanoporous alumina with the aim to correlate measured capacitance changes with specific binding of biomarker proteins. The second contribution by Maguire et al. aims to address the requirement for improved prediction of pharmacokinetics and pharmacodynamics profiles of candidate drugs before clinical evaluation that would ultimately decrease the currently high late-stage failure rates upon completion of Phase-I clinical trials due to human toxicity or bioavailability problems. The authors provide a computational fluid dynamics approach that successfully models experimental observation of increases in the clearance rates of high- and medium-clearance compounds in microfluidic systems. Based on their findings they propose a general effect of convective transport in compound clearance attributed to the thinning of the boundary layer present in the static and mixed plate culture systems relative to microfluidic systems that seem to provide better correlations.

This issue contains four contributions in the area of therapeutics and drug delivery. Mourtas et al. describe the design and evaluation of liposomal gels for the vaginal delivery of the microbicide MC-1220 that is a highly potent and selective non-nucleoside reverse transcriptase inhibitor of HIV. Sharma et al. describe an oligonucleotide functionalized nanoparticle approach that aims to specifically target the scavenger receptors on macrophage foam cells with the ultimate goal to therapeutically intervene in the progression of atherosclerosis leading to plaque rupture and thrombosis. Swanson and Borden describe their approach for injectable oxygen delivery that may be proven indispensable in the critical care field for maintaining oxygen supply to hypoxia-sensitive brain and heart tissue. Their approach involves albumin-based microbubbles encapsulating oxygen that are specifically designed for intravenous injection. Finally, Jain et al. contribute an informed, timely review on 
non-condensing polymeric gene delivery systems; they describe the design principles and discuss potential applications.

Introduction of multiple functionalities on nanometer-sized particles is expected to lead to a new chapter in nanotechnology for nanomedicine. Nikitczuk et al. study the stimulation of dendritic cells by a multi-faceted nanopolymer-based delivery system with the aim to understand the complex stimulation mechanisms that may ultimately be exploited in an immunotherapeutic setting. Huth et al. describe the design and characterization of superparamagnetic nanoparticles coated with phospholipid layers that are loaded with hydrophobic drug analogues. The authors utilize the heating properties of nanoparticles in the presence of an alternating magnetic field to induce fast release of the drug surrogate from the heated lipid layer. Boyes et al. review the area of nanoscale diagnostic systems that incorporate imaging agents and, in particular, the chemical aspects in the development of molecular probes based on gadolinium nanoparticles. These systems are emerging as the next generation imaging agents, and, therefore, the discussion on their potential role in translational clinical imaging and therapy is particularly timely. Lin et al. explore the potential of polymer-coated plasmonic gold nanorods for photothermal ablation of cancer in a genetically engineered mouse model that more accurately recapitulates the human disease in terms of structure and biology relative to subcutaneous xenograft models. And finally, Huang et al. review the recent progress reported in the imaging, photothermal therapy and gene/drug delivery using plasmonic gold nanoparticles of various forms.

This issue on nanomedicine concludes with a feasibility study by Marquet et al. on opening the blood-brain barrier using a clinical ultrasound system which has the potential to impact drug delivery strategies to the brain.

We hope this issue on nanomedicine to inform and inspire Nano LIFE's readers.

Fall 2011

Stavroula Sofou, Ph.D.

Kaushal Rege, Ph.D. 